\title{
Using Seed Chipping to Genotype Maize Kernels
}

Alison M. Mills, Lindy A. Allsman, Sareen Leon and Carolyn G. Rasmussen*

Department of Botany and Plant Sciences, University of California, Riverside, California 92521, USA

*For correspondence: carolyn.rasmussen@ucr.edu

\begin{abstract}
[Abstract] Zea mays (maize) is an important model organism for studying monocot growth and development. Genotyping maize in the greenhouse or field can be time consuming and costly. Here, we describe a method to remove or chip a small amount of the endosperm from a maize kernel to genotype the kernel prior to planting. The seed chip is removed with a razor blade for DNA extraction and subsequent genotyping. When done correctly, seeds germinate normally and the kernel genotype can be determined before planting thus saving time, money, and field space.
\end{abstract}

Keywords: Zea mays, Maize, Seed chipping, Kernel chipping, Genotyping

[Background] Maize is an agriculturally important crop that is grown throughout the world. It has many uses including human consumption, animal feed, and ethanol production (Ranum et al., 2014). Maize is also a useful model for investigating a wide range of biological questions including plant domestication, monocot growth and development, pest resistance, and genome evolution (Strable and Scanlon, 2009). Although maize has many scientific and industrial uses, it is a large plant that requires significant space in either a greenhouse or field to grow. In addition to the cost of maintaining a greenhouse or field, considerable effort is required to perform directed crosses and collect plant material for genotyping and other analyses. Due to these factors, the ability to genotype maize kernels prior to planting is valuable. This protocol describes the process of seed chipping, where a small piece of endosperm is removed with a razor blade to be used for DNA extraction and genotyping. This protocol is a modification of a method where kernels are soaked in water prior to chipping (Gao et al., 2008). We use fully dried maize kernels for chipping to prevent problems we encountered using pre-soaked kernels such as fungal contamination and unanticipated germination. This method is also useful when genotyping a large number of kernels, because the dry kernel chips do not have to be processed immediately for DNA extraction.

Chipping seeds eliminates costs associated with leaf genotyping. Growing maize plants in a field to collect leaf material takes time, labor, and money. These factors make seed chipping cost an estimated $24.6 \%$ lower than leaf genotyping in the field (Gao et al., 2008). Seed chips can also be stored at room temperature, unlike leaf tissue which needs to be kept cool and wet in a refrigerator or dried prior to storage at room temperature.

A major advantage of seed chipping the ability to select specific genotypes before planting. Determining the genotype of seeds prior to planting allows for more efficient field planning. This technique is particularly valuable for marker-assisted selection, which relies on molecular markers associated with important crop traits (Xu et al., 2008). Seed chipping has the potential to reduce the cost 
and increase the efficiency of such breeding programs. We demonstrate here that germination rates are nearly identical between chipped and unchipped seeds.

\section{Materials and Reagents}

1. Tungsten carbide beads (diameter $3 \mathrm{~mm}$ ) (Qiagen, catalog number: 69997)

2. Straight edge razor blades

3. $2.0 \mathrm{ml}$, Safe-Lock microcentrifuge tubes (Eppendorf, catalog number: 022363352)

4. 6-quart plastic storage containers or similar sized plastic container

5. Paper towels

6. 96-well microcentrifuge tube rack (Genesee Scientific, catalog number: 27-119)

7. 48-well tissue culture plates (VWR, catalog number: 10062-898)

8. Zea mays (maize)

9. $70 \%$ ethanol

10. Water

\section{Equipment}

1. Forceps (Fine Science Tools, catalog number: 91150-20)

2. TissueLyser (Qiagen, TissueLyser II, catalog number: 85300)

3. TissueLyser Adapter Set $2 \times 24$ (Qiagen, catalog number: 69982)

\section{Procedure}

A. Labeling tubes and plates

1. Label the bottom of a 48-well tissue culture plate with relevant information such as family number, parent genotypes, date, etc. and label each well 1-48 (Figure 1A). The plate should also be labeled with a short unique ID such as a letter, initials, or a number. This unique ID should be short enough to fit on the lid of a $2 \mathrm{ml}$ microcentrifuge tube. Kernels will be stored in this plate after chipping.

2. Place 48 microcentrifuge tubes in a 96-well microcentrifuge tube rack. Label each tube with the corresponding well number and short plate ID (Figure 1B). The individual kernel chips will be placed in these tubes. 


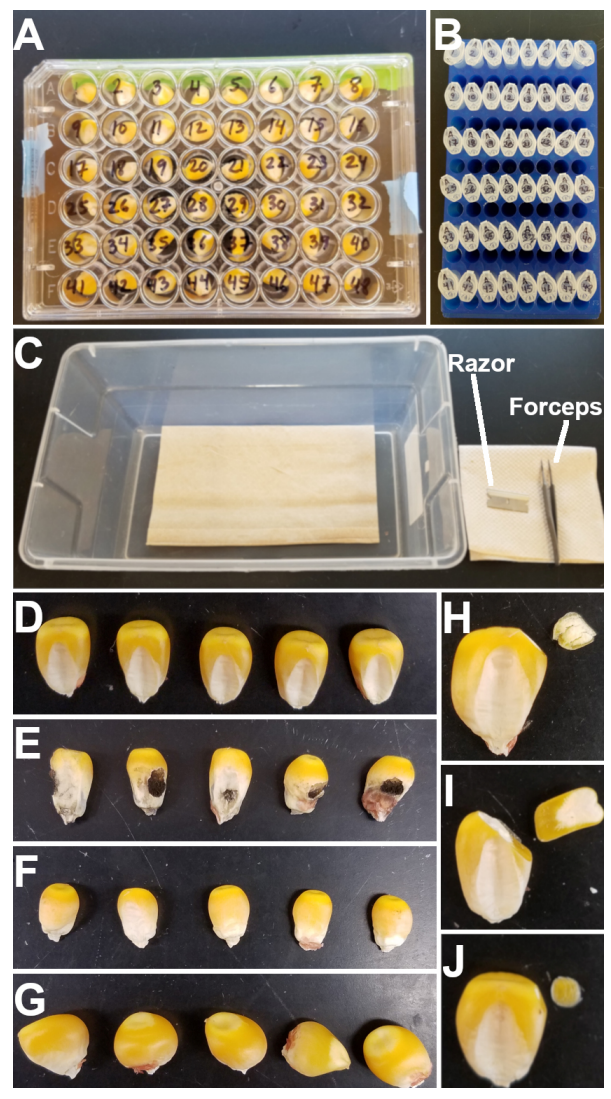

Figure 1. Set up for seed chipping. A and B. Labeling plates and tubes for seed chipping. A. A 48-well plate labeled for storage of chipped kernels. Plates should be labeled on the side or bottom with any relevant information such as parent genotypes, family number, date. A short ID may also be written on the plate. B. $482 \mathrm{ml}$ microcentrifuge tubes arranged in a 96-well microcentrifuge tube rack to store seed chips. Labels on each tube correspond to the wells of the labeled 48 well plate in panel A. In this example, the plate was given the letter "A" as a short ID that could easily fit on the lids of the $2 \mathrm{ml}$ tubes. C. Typical set up for seed chipping kernels for genotyping. A straight razor is used to remove a small piece of the kernel. Forceps are used to transfer the kernel chip and chipped kernel to a $2 \mathrm{ml}$ tube and plate well respectively. Chipping within a small plastic box helps prevent chips from being lost. D-G. Examples of typical kernels. D. Examples of kernels ideal for chipping, with large regular shapes and free of damage. E. Examples of kernels that should not be chipped due to damage from mold. F. Examples of kernels that are difficult to chip due to their small size. G. Examples of round kernels that are difficult to grip and should be chipped carefully. H-J. Examples of chip sizes. H. A typical size kernel chip that is sufficient for genotyping without damaging the embryo. I. A kernel chip that is too large and may potentially damage the embryo. J. A kernel chip that is too small to yield sufficient quantities of DNA for genotyping. 


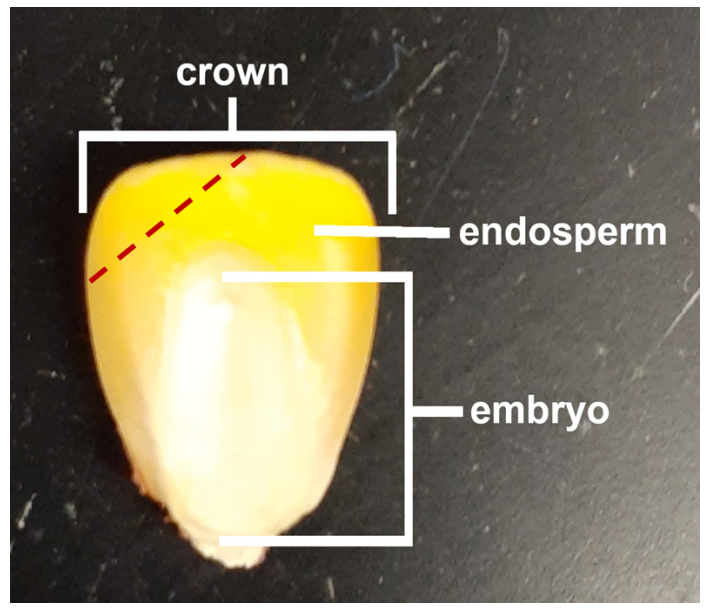

Figure 2. Anatomy of a maize kernel. The region containing the maize embryo is in the pale indented area near the pointed end of the kernel. The endosperm is located in the areas surrounding the embryo. To avoid damaging the embryo while chipping, chips should be taken from the broad crown of the kernel. The dashed red line indicates an acceptable site to chip the kernel, which will yield enough endosperm to extract DNA for genotyping without damaging the embryo.

B. Chipping kernels

1. Place a dry paper towel inside a clean 6-quart plastic tub (Figure 1C). Chipping is done in a tub to prevent losing the seed chip during cutting.

2. Place a second paper towel outside of the tub and moisten it with $70 \%$ ethanol. This paper towel will be used to wipe off kernel debris from the razor and forceps during the chipping process.

3. Place a single dry kernel in the plastic tub on top of the dry paper towel.

Note: Kernels should be in good condition, and free of mold or insect damage (Figures 1D and $1 E)$.

4. While wearing gloves, securely grip the end of the kernel containing the embryo with one hand (Figure 2). With the other hand, use a sharp straight edge razor blade to carefully slice a small piece of endosperm from the crown of the kernel (Video 1). Take care to avoid cutting into the kernel embryo (Figures $1 \mathrm{H}-1 \mathrm{~J}$ ).

Note: A small chip should be sufficient (Figure 1H). A small amount of white, starchy endosperm should be visible on the chip. If only hard, yellow pericarp is present, the chip may be too small to acquire sufficient DNA for genotyping (Figure 1J). However, cutting too large a piece or cutting the embryo may prevent the kernel from germinating (Figure 1I). 


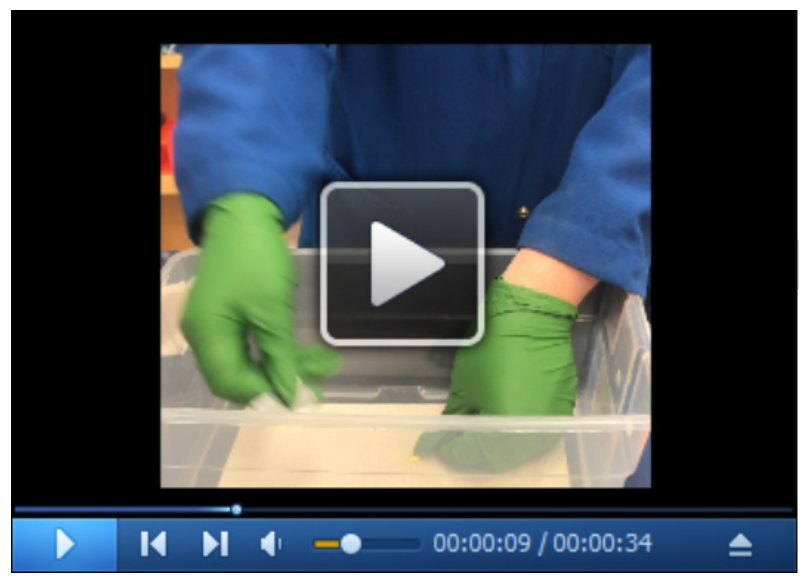

Video 1. Chipping maize seed with a razor blade

5. Use a clean pair of forceps to place the chip into a previously labelled $2 \mathrm{ml}$ microcentrifuge tube. Next, use the forceps to place the kernel into its respective well in the 48-well plate.

6. Wipe the razor and forceps clean on the paper towel moistened with $70 \%$ ethanol.

7. Shake any kernel residue off the dry paper towel where the kernel was chipped. The plastic tub should be wiped down if residue from the previously chipped kernel is present.

8. Repeat Steps B3-B7 for each individual kernel to be genotyped.

9. The collected chips should be stored with the tube caps opened to prevent fungal growth. Chips should be ground immediately prior to DNA extraction. The kernels should be stored with the plate lid on (preferably taped in place to prevent spills) in a dry, room temperature environment. The chipped kernels can be stored for up to a month without any significant loss of germination, and good quality DNA can be acquired from seeds less than 2 years old stored at room temperature (Gao et al., 2008). The effects of seed chipping on germination over a longer timescale have not been closely examined. 


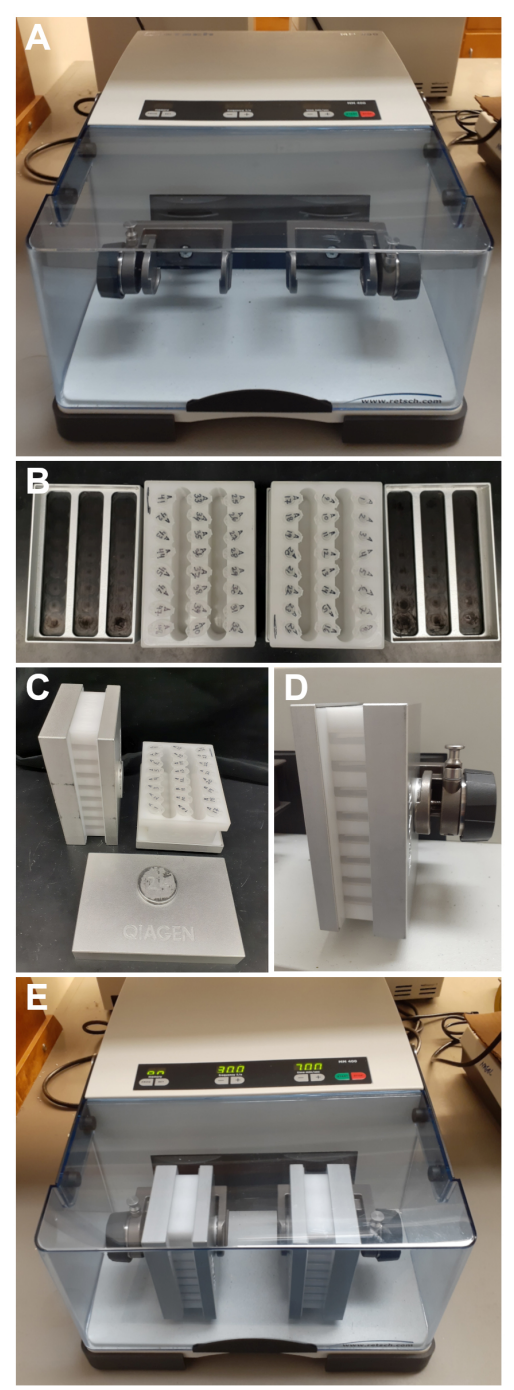

Figure 3. How to operate a TissueLyser. A. An empty TissueLyser. B. $482 \mathrm{ml}$ microcentrifuge tubes containing seed chips and tungsten carbide beads loaded into two 24-tube adapters. C. A fully assembled adapter (left) and an open adapter (right). D. Assembled adapter properly positioned so circular elevations on the adapter are flush with the arm of the Tissuelyser. The adapter is secured in place by turning the dark gray dials to tighten the TissueLyser arm. The adapters should be securely held in place so they do not slip, but care should be taken not to overtighten the TissueLyser arms. E. TissueLyser with adapters and samples ready to run.

C. Tissue grinding

1. Place a $3 \mathrm{~mm}$ tungsten carbide bead into each $2 \mathrm{ml}$ microcentrifuge tube containing a kernel chip.

Note: Be mindful when adding beads and closing lids on tubes because these movements can jolt the tubes, causing seed chips to jump. This can result in loss of seed chips or contamination of other tubes with different seed chips, potentially resulting in incorrect genotyping results. Carefully close all the tubes in the rack, and then add the beads to each tube individually. Each tube is then closed after the bead is added to prevent contamination by chips from nearby tubes. 
2. Close the tubes securely and place them into Qiagen adapters for the TissueLyser (Figure 3A). 48 tubes can be ground at one time using two 24-tube adapters (Figures $3 \mathrm{~B}$ and $3 \mathrm{C}$ ). If grinding using fewer tubes, make sure each adapter has an equal number of tubes so the weight of each adapter is equal. Set the time to $7 \mathrm{~min}$ at a frequency of 30 strokes per minute.

Note: Follow Qiagen manufacturer guidelines for correct, safe use of the TissueLyser.

3. Place the adapters into the TissueLyser (Figures $3 \mathrm{D}$ and $3 \mathrm{E}$ ). Secure them in place but do not overtighten the screws. Make sure the circular elevations on the adapters are flush with the arms of the TissueLyser. Run the samples.

Note: If large pieces of unground tissue remain after grinding, the adapters can be removed and rotated 180 degrees. The program can then be run again to grind the tissue further.

4. Proceed to extract DNA and genotype.

Note: We recommend a protocol such as "Small-scale DNA Extraction Method for Maize and Other Plants" (Lunde, 2018). Other methods of DNA extraction can also be used successfully, including other common methods for plant DNA extraction such as the "NaOH-Tris method" (von Post et al., 2003) or commercially available plant DNA extraction kits.

\section{Notes}

1. Potentially challenging kernels

The size of the chip that can be safely taken from a kernel may vary based on the size and shape of the kernel. Extra care should be taken with round kernels. These kernels are harder to grip and pose a higher risk of slipping and accidentally cutting the embryo or fingers (Figure 1G). Smaller chips should be taken from small kernels (Figure 1F). However, it is possible to remove too little tissue (Figure $1 \mathrm{~J}$ ). If too little tissue is removed from a kernel, there may be insufficient DNA extracted to genotype the kernel. Moldy or damaged kernels should not be used for seed chipping (Figure 1E). Damaged kernels tend to lack sufficient healthy tissue to chip and have reduced viability.

2. Concerns about germination

Seed chipping does not significantly alter the rate of germination (Table 1). However, if it is suspected that chipping may be influencing germination, comparison of chipped and unchipped germination rates can be assessed by germinating chipped and unchipped kernels side by side on soil or moist paper towels. This comparison can rule out other potential causes of poor germination such as old seeds, improper storage and handling, or poor planting conditions. 
Table 1. Germination rates of chipped versus unchipped kernels for 3 separate families of B73 kernels. 30 kernels were chipped for each family and then germinated on moist paper towels alongside 30 unchipped kernels from the same family for comparison. Germination was assessed 6 days later. Chipping did not affect germination rate.

\begin{tabular}{ccc}
\hline Family & Unchipped & Chipped \\
\hline 1 & $27 / 30$ & $28 / 30$ \\
2 & $30 / 30$ & $30 / 30$ \\
3 & $30 / 30$ & $30 / 30$ \\
\hline
\end{tabular}

3. Possible sources of contamination

There are a few potential sources of contamination specific to seed chipping that can cause genotyping errors: hetero-fertilization and pericarp contamination. In maize, hetero-fertilization can occur when two different male gametes fertilize one egg. This can result in one male gamete forming the embryo and the other one forming the endosperm. Alternatively, the egg and polar nuclei may have different genotypes even though they fuse with identical sperm. However, hetero-fertilization in maize is infrequent and occurs at an average rate of 1.25\% (Sprague, 1932). A skewed ratio of expected genotypes versus those obtained by genotyping may indicate hetero-fertilization has occured. In such cases extracting DNA from leaves should be used instead of seed chipping.

Pericarp contamination can occur when DNA from the pericarp, a maternally derived tissue, on the chip interferes with genotyping. This can be a problem in cases where the maternal genotype differs from the embryo genotype. However, usually the small amount of pericarp on the chip does not interfere with genotyping. Pericarp contamination can be identified by comparing the expected ratio of genotypes in the progeny to the genotyping results obtained. A skewed ratio of genotypes in the progeny may indicate pericarp contamination is present. Possible solutions include designing new primers or using DNA extracted from leaves for genotyping (Gao et al., 2008). The pericarp could also be carefully removed from the chip with a razor or scalpel, but this not recommended as it is a labor-intensive and difficult process.

\section{Acknowledgments}

CGR gratefully acknowledges NSF-MCB 1716972 for funding, and authors thank Alexander Noriega (University of California, Riverside) for beta testing this protocol and helpful comments. The authors declare no conflict of interest. This protocol was modified from Gao et al. (2008). 


\section{$\underline{\text { References }}$}

1. Gao, S., Martinez, C., Skinner, D. J., Krivanek, A. F., Crouch, J. H. and Xu., Y. B. (2008). Development of a seed DNA-based genotyping system for marker-assisted selection in Maize. Molecular Breeding: New Strategies in Plant Improvement 22 (3): 477.

2. Lunde, C. (2018). Small-scale DNA extraction method for maize and other plants. Bio-101: e2782.

3. von Post, R., von Post, L., Dayteg, C., Nilsson, M. and Tuvesson, S. (2003). A high-throughput dna extraction method for barley seed. Euphytica 130 (2): 255-260.

4. Ranum, P., Peña-Rosas, J. P. and Garcia-Casal, M. N. (2014). Global maize production, utilization, and consumption. Ann N Y Acad Sci 1312: 105-112.

5. Sprague, G. F. (1932). The nature and extent of hetero-fertilization in maize. Genetics 17(3): 358-368.

6. Strable, J. and Scanlon, M. J. (2009). Maize (Zea mays): a model organism for basic and applied research in plant biology. Cold Spring Harb Protoc 2009(10): pdb emo132.

7. Xu, Y. B. and Crouch., J. H. Crouch. (2008). Marker-assisted selection in plant breeding: from publications to practice. Crop Sci 48: 391-407. 